\title{
Stars and gas in the Medusa merger ${ }^{\star}$
}

\author{
E. Manthey ${ }^{1,2}$, S. Hüttemeister ${ }^{2}$, S. Aalto ${ }^{3}$, C. Horellou ${ }^{3}$, and P. Bjerkeli ${ }^{3}$
}

1 Astron, 7990AA Dwingeloo, The Netherlands

e-mail: juette@astron.nl

2 University of Bochum, Department of Astronomy, 44780 Bochum, Germany

3 Onsala Space Observatory, Chalmers University of Technology, 43992 Onsala, Sweden

Received 17 June 2008 / Accepted 20 August 2008

\section{ABSTRACT}

\begin{abstract}
The Medusa (NGC 4194) is a well-studied nearby galaxy with the disturbed appearance of a merger and evidence for ongoing star formation. In order to test whether it could be the result of an interaction between a gas-rich disk-like galaxy and a larger elliptical, we have carried out optical and radio observations of the stars and the gas in the Medusa, and performed $N$-body numerical simulations of the evolution of such a system. We used the Nordic Optical Telescope to obtain a deep $V$-band image and the Westerbork Radio Synthesis Telescope to map the large-scale distribution and kinematics of atomic hydrogen. A single HI tail was found to the South of the Medusa with a projected length of $\sim 56 \mathrm{kpc}\left(\sim 5^{\prime}\right)$ and a gas mass of $7 \times 10^{8} M_{\odot}$, thus harbouring about one third of the total HI mass of the system. HI was also detected in absorption toward the continuum in the center. HI was detected in a small nearby galaxy to the North-West of the Medusa at a projected distance of $91 \mathrm{kpc}$. It is, however, unlikely that this galaxy has had a significant influence on the evolution of the Medusa. The simulations of the slightly prograde infall of a gas-rich disk galaxy on an larger, four time more massive elliptical (spherical) galaxy reproduce most of the observed features of the Medusa. Thus, the Medusa is an ideal object to study the merger-induced star formation contribution from the small galaxy of a minor merger.
\end{abstract}

Key words. galaxies: interactions - galaxies: starburst - galaxies: individual: NGC 4194 - radio lines: galaxies - radio lines: ISM

\section{Introduction}

The Medusa galaxy (NCG 4194, Arp 160, IZw33) is a nearby galaxy $(D=39 \mathrm{Mpc})$ with the disturbed appearance typical of a merger (see Fig. 1). It has been interpreted as a candidate for a minor merger between a large elliptical and a small spiral galaxy, a so-called S+E merger (Aalto \& Hüttemeister 2000). The galaxy earned its nickname from the fuzzy and knotty optical tail to the North, reminiscent of hair or tentacles. A sharp shell-like structure is visible on the opposite side of the galaxy, confining the optical main body. A second, fainter shell can be seen further out on deep images (e.g., Jütte et al. 2008).

Major mergers between two gas-rich disk galaxies can lead to ultraluminous infrared galaxies (ULIRGs, $L_{\mathrm{FIR}}>10^{12} L_{\odot}$ ) due to an extreme increase of star formation (Sanders et al. 2003). Strong gas inflows are triggered in these mergers, increasing the gas density in the centre and thus leading to the observed starburst (e.g., Barnes \& Hernquist 1991, 1996; Mihos \& Hernquist 1996). In these simulations the merger remnant evolves into a regular elliptical galaxy. In contrast, in minor, i.e., unequal-mass mergers only a moderate, if at all, starburst is induced implying a lower far-infrared (FIR) luminosity (less than $\left.10^{11} L_{\odot}\right)($ Cox et al. 2008). The triggered star formation and the remnant galaxy type strongly depend on the mass ratio of the merging pair, but not so much on the total gas mass (Bournaud et al. 2005; Cox et al. 2008). In contrast to most nearby minor mergers, in which the major galaxy is a spiral and thus dominating the star formation (e.g., M 51), the Medusa gives us an ideal opportunity to investigate the influence of the merger on the smaller companion because it is the only gas-rich partner here.

\footnotetext{
* Movies are only available in electronic form at http: //www . aanda.org
}

Since the merger-induced star formation does not depend on the gas content of the companion (Martig \& Bournaud 2008), the results of the Medusa might be applicable to other minor mergers, independent on the type of the large partner.

Another significant difference between ULIRGs and galaxies like the Medusa is the extent of the starburst induced by the merger. While the burst region in ULIRGs is compact and typically concentrated to the inner kiloparsec, (e.g., Sanders \& Mirabel 1996), the Medusa exhibits an extended region of ongoing star formation which is, however, not as intense as in ULIRGs (Armus et al. 1990; Prestwich et al. 1994). Weistrop et al. (2004) analyzed optical and ultraviolet Hubble Space Telescope (HST) images of the nucleus and found star-forming knots younger than $20 \mathrm{Myr}$ in the center. They derived a star formation rate (SFR) of $\sim 6 M_{\odot} \mathrm{yr}^{-1}$, but argued that the overall SFR in the Medusa might be as high as $30 M_{\odot} \mathrm{yr}^{-1}$ since the knots produce only $20 \%$ of the UV flux in the center. Based on recent HST spectroscopic observations, Hancock et al. (2006) estimate an even higher total $S F R$ of $\sim 46 M_{\odot} \mathrm{yr}^{-1}$, and ages of individual star forming regions of 5.5-10.5 Myr.

The Medusa possesses a reservoir of gas, both molecular and atomic. High resolution aperture synthesis maps obtained with the Owens Valley Radio Observatory revealed extended $\mathrm{CO}$ emission on a total scale of $25^{\prime \prime}$ (4.7 kpc) (Aalto \& Hüttemeister 2000). The CO morphology is complex, occupying mainly the center and the north-eastern part of the main optical body. The extended $\mathrm{CO}$ gas follows two prominent dust lanes: one which is crossing the central region at right angle with respect to the optical major axis, and a second which curves to the north-east and into the beginning of the northern optical tail. Aalto \& Hüttemeister (2000) suggested that the central starburst is being fueled by gas flows along the central dust lane. The 
presence of multiple spectral peaks $\left(\Delta V \approx 200 \mathrm{~km} \mathrm{~s}^{-1}\right)$ suggests that at least two velocity systems coexist in the $\mathrm{CO}$ emitting gas. Either this is an effect of substantial warping of the main body, or simply the unrelaxed, overlapping systems of the progenitor galaxies. The multiple features seem to be related to the extended dust lanes, but several velocity components co-exist also at the base of the optical tail. Single-dish measurements revealed the presence $\mathrm{CO}$ emission even inside the optical tail out to $4.7 \mathrm{kpc}$ away from the center (Aalto et al. 2001) where there is no sign of ongoing star formation (Armus et al. 1990). The total molecular gas mass in the inner $2 \mathrm{kpc}$ was estimated to $2 \times$ $10^{9} M_{\odot}$. Thus, the star formation efficiency is almost as high as in ULIRGs although the molecular gas density is significantly lower (Aalto \& Hüttemeister 2000). Besides uncertainties in the molecular gas conversion factor, this gives a hint that the ISM in these mergers is different compared to the molecular cloud complexes in normal galaxies and also in extreme starbursts. Aalto \& Hüttemeister (2000) argue that the observed solid-body rotation may offer a shear-free environment. This may support cloud collapse and thus star formation. The star formation rate might have increased because a large amount of molecular gas was transported to the centre on short time scales by the interaction.

The galaxy contains a similar amount of atomic gas as molecular gas $\left(M_{\mathrm{HI}} \sim 2.2 \times 10^{9} M_{\odot}\right)$, inferred from the singledish HI emission spectrum obtained by Thuan \& Martin (1981) using the Greenbank $91 \mathrm{~m}$ telescope. Atomic gas has recently been detected in absorption toward the center of the Medusa, through sub-arcsecond MERLIN observations (Beswick et al. 2005). The kinematics of the HI gas in the central region of the galaxy is similar to that of the molecular gas traced by the CO emission (Aalto \& Hüttemeister 2000), which suggests that the atomic and molecular component of the cold interstellar medium are probably physically related. Two compact radio sources were found in the central parsec, embedded in a region of diffuse radio continuum emission. Even though the presence of a weak AGN cannot be ruled out completely, most (if not all) of the continuum emission is related to the starburst (Beswick et al. 2005).

NGC 4194 displays conflicting evidence about its merger age. The sub-arcsecond resolution MERLIN data revealed two compact radio components in the center, separated by $\sim 0 ! 35$, corresponding to $65 \mathrm{pc}$ only (Beswick et al. 2005). The optical tail is fairly diffuse, which is suggestive of an advanced merger where the progenitors have coalesced. In contrast, the fairly widespread $\mathrm{CO}$ distribution, irregular optical isophotes, multiple spectral $\mathrm{CO}$ features, and the distorted central body hint toward a younger system.

In this paper, we present observations and $N$-body numerical simulations of the stars and the gas in the Medusa merger. We mapped the large-scale distribution and kinematics of the HI gas with the Westerbork Radio Synthesis Telescope (WSRT) and used the Nordic Optical Telescope (NOT) to obtain a deep $V$-band image of the distribution of the stars in the Medusa. Then we carried out numerical simulations which we compared to the observations. HI $21 \mathrm{~cm}$ line observations are a powerful tool to trace the dynamical history of interacting and merging galaxies and provide constraints to dynamical models of the history of the interaction. They complement $\mathrm{CO}$ millimeter-wave line observations that trace mainly the inner part of galaxies where the molecular gas resides. Furthermore, they offer is the only way to trace the distribution and kinematics of gas in large scale tidal features. The observations are presented in Sects. 2 and 3, and the simulations in Sect. 4. Follows a discussion.
Table 1. Basic properties of NGC 4194. The distance is based on $H_{0}=$ $75 \mathrm{~km} \mathrm{~s}^{-1} \mathrm{Mpc}^{-1}$.

\begin{tabular}{lc}
\hline \hline RA $(2000)$ & 121409.5 \\
Dec $(2000)$ & +543137 \\
$v_{\text {hel,opt }}\left(\mathrm{km} \mathrm{s}^{-1}\right)$ & 2442 \\
$D(\mathrm{Mpc})$ & 39 \\
$L_{\mathrm{B}}\left(10^{9} L_{\odot}\right)$ & 15.7 \\
$L_{\text {FIR }}\left(10^{10} L_{\odot}\right)$ & 8.5 \\
FIR flux at $60 \mu \mathrm{m}(\mathrm{Jy})$ & 25.66 \\
FIR flux at $100 \mu \mathrm{m}(\mathrm{Jy})$ & 26.21 \\
\hline
\end{tabular}

Table 2. Observing parameters of the WSRT observations.

\begin{tabular}{lc}
\hline \hline Observing parameters & \\
\hline Date & 12.7 .2003 \\
Central frequency & $1408.6 \mathrm{MHz}$ \\
Total bandwidth & $20 \mathrm{MHz}$ \\
Number of channels & 256 \\
Velocity resolution & $4.12 \mathrm{~km} \mathrm{~s}^{-1}$ \\
Synthesised beamwidth & $23^{\prime \prime} \times 19^{\prime \prime}$ \\
Primary beam & $36^{\prime}$ \\
Primary calibrator & $3 \mathrm{C} 147$ \\
\hline
\end{tabular}

\section{Observations and data reduction}

\subsection{HI observations}

The observations were carried out with the WSRT in July 2003. We observed one full $12 \mathrm{~h}$ track in the maxi-short array configuration under good conditions. As a flux calibrator, the standard source 3C 147 was observed before and after the target. No phase calibrator was observed, since the standard procedure at WSRT for phase calibration is to obtain self-calibration. In Table 2 we list the observing parameters and give information about the setup used. We chose parameters which allowed us to both cover a large velocity range, because HI lines can typically be broader in mergers than in normal disk galaxies, and to reach a high resolution in velocity to be able to detect narrow features.

The data were reduced using MIRIAD together with additional, WSRT specific routines written by Oosterloo ${ }^{1}$. The quality of the data was good, so that only little flagging of interferences was necessary. First, the data had to be calibrated to system temperature scale. Since no secondary calibrator was observed, after flux and bandpass calibration using the primary calibrator, a continuum image was created to perform self-calibration on continuum sources within the field. To achieve a deep CLEANing, we iteratively created a mask which includes emission only and which defines the regions to be CLEANed. After CLEANing and self-calibration a new mask was created and the process repeated until no improvement could be made. The results were then applied to the target galaxies.

CLEANing the HI cube was done in a similar way to CLEANing the continuum. After continuum subtraction, we applied Hanning smoothing, since this was not done during the observations. Again, we used as mask containing emission only to define CLEANing boxes. This procedure was repeated 3 times until the noise level was reached. Integrated intensity and velocity maps were created using the MOMENT task in MIRIAD. Finally, primary beam correction was applied to all data.

We produced two sets of maps: naturally weighted ones (with an rms noise level of $0.4 \mathrm{mJy}^{\text {beam }}{ }^{-1}$, resolution of $31^{\prime \prime} \times$ $27^{\prime \prime}$ ), and maps obtained using a ROBUST factor of 0.5 , which

1 http://www.astron.nl/ oosterlo/wsrtMiriad/ 

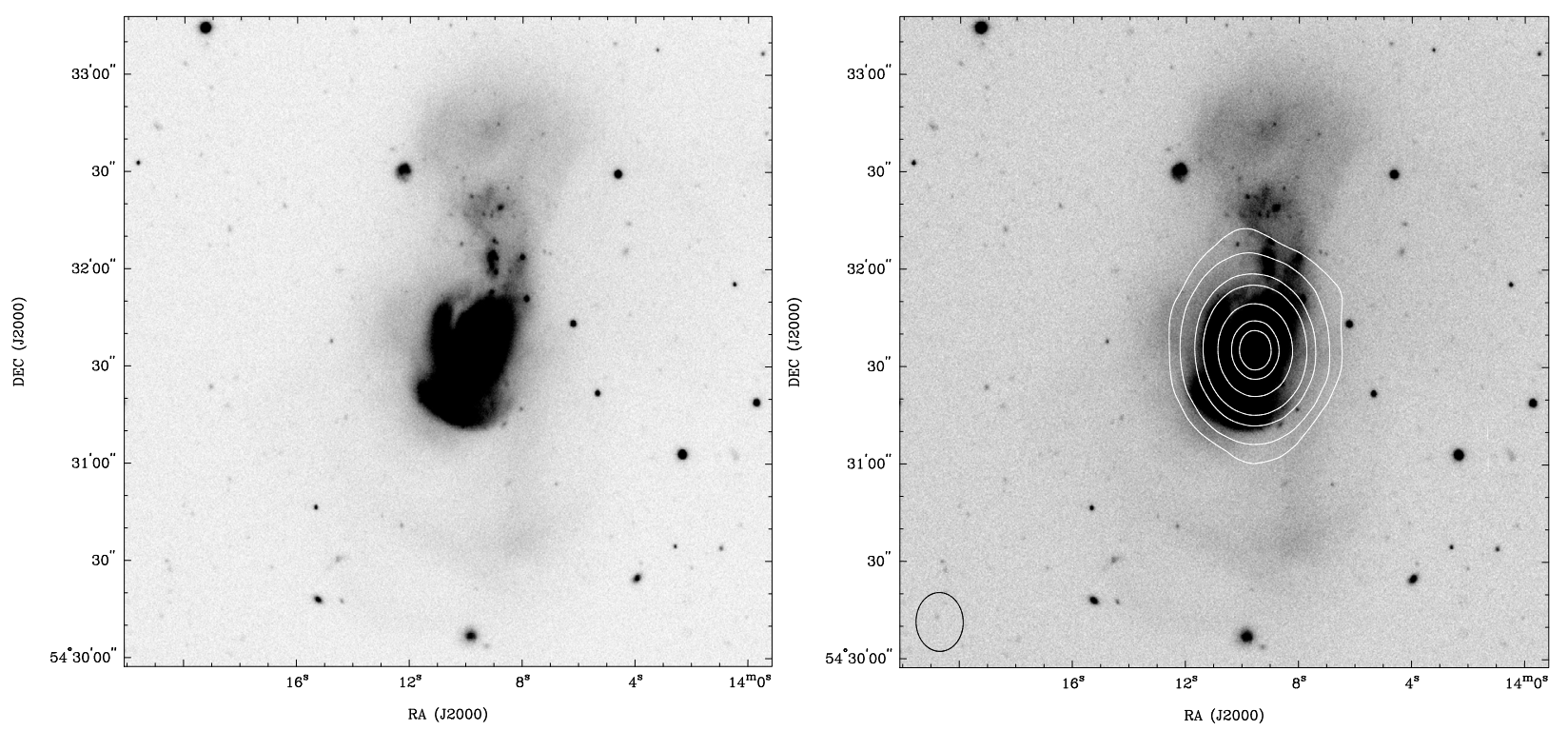

Fig. 1. Left: nordic Optical Telescope $V$-band image of the Medusa. Right: contour map of the $20 \mathrm{~cm}$ continuum emission superimposed on the $V$-band image. The synthesised beam is shown in the lower left corner. The contour levels are $0.2,0.5,2,5,20,50,70 \mathrm{mJy} \mathrm{beam}^{-1}$.

gives a better resolution with slightly less sensitivity (noise level of $0.6 \mathrm{mJy}_{\text {beam }}{ }^{-1}$, resolution of $\left.23^{\prime \prime} \times 19^{\prime \prime}\right)$. Since the morphological features were the same in both sets of maps, we present here only the ROBUST 0.5 maps in which structures in the HI tail appear more clearly.

\subsection{Optical imaging}

We also present here optical Johnson $V$-band images taken with ALFOSC mounted at the Nordic Optical Telescope (NOT) on La Palma, Canary Islands in January 2003. The field of view of ALFOSC is 6. $.4 \times 6.4$, thus large enough to cover not only NGC 4194 completely but also a sufficient region of sky for a proper background subtraction. The integration time was $10 \mathrm{~min}$. Standard reduction was applied to the data using the IRAF software. A further description of the observations and reduction as well as a detailed analysis of the optical data will be given in Jütte et al. (2008).

\section{Observational results}

\subsection{Radio continuum}

Figure 1 shows the NOT $V$-band image of NGC 4194 on which contours of the $20 \mathrm{~cm}$ radio continuum emission have been superimposed. The flux density of the source is $0.108 \mathrm{Jy}$. We see continuum emission extending out to a deconvolved size of 6.' 6 $(1.2 \mathrm{kpc})$ associated with the galactic main body. This is not surprising, since the $20 \mathrm{~cm}$ continuum flux is a tracer of star formation and NGC 4194 exhibits an extended region of intense star formation. The continuum map shows a smooth distribution center at the position of the optical nucleus.

\subsection{Neutral hydrogen}

Figure 2 shows the integrated HI intensity map overlayed on an optical image from the Digitized Sky Survey (DSS). HI gas is found all over the optical extent of the Medusa. Remarkable is the single long HI tail to the South. HI is also found in absorption toward the center. We also detect HI associated with the small nearby galaxy SDSS J121326.03+543631.7 identified in the Sloan Digitized Sky Survey about 8' to the north-west.

The HI properties of NGC 4194 and of the nearby dwarf galaxy are summarised in Table 3. The integrated HI spectra are shown in Fig. 3. We calculate a total HI mass of NGC 4194 of $2 \times 10^{9} M_{\odot}$, in good agreement with the HI mass determination by Thuan \& Martin (1981) of $2.2 \times 10^{9} M_{\odot}$. This shows that our interferometric observations do not miss any extended emission.

\subsubsection{The main body of NGC 4194}

Figure 4 shows the HI distribution, the velocity field and the velocity dispersion map. The channel maps are shown in Fig. 5. Figure 6 shows the HI integrated emission as isocontours overlaid on our optical $V$-band image, which is deeper than the DSS image but covers only the upper part of the HI tail. The HI emission peaks north of the absorption feature, aligned with the beginning of the optical tidal tail and some bright knots, which might be proto-star clusters. The HI embeds the optical tail completely, but is not considerably extended further out, i.e., no pronounced HI tail as a counterpart of the optical tail is found. The optical tail seems to be embedded in a smooth HI distribution somewhat extended towards the north-west, maybe coincidentally in the direction where the possible companion is found.

In the optical, we see two shells in the south, a bright one close to the center and a second, fainter one at a larger radius. We do not see any feature, neither in the HI distribution nor in the velocity and velocity dispersion maps which is related to these shells.

The velocity map (Fig. 4, bottom left) shows a slightly disturbed rotating disk, represented by a typical spider diagram, with regions of higher velocity on the eastern side and lower velocity on the western side on the main body of the Medusa.

The HI velocity dispersion is larger in the galactic body than in the tail. The highest values are measured north-east of the absorption region, where there is a slight dip in the HI distribution. This region is also roughly related to the dust lane where extended molecular gas was found (Aalto \& Hüttemeister 2000). In the molecular gas maps multiple velocity components are found, mimicing high velocity dispersion in an integrated dispersion 


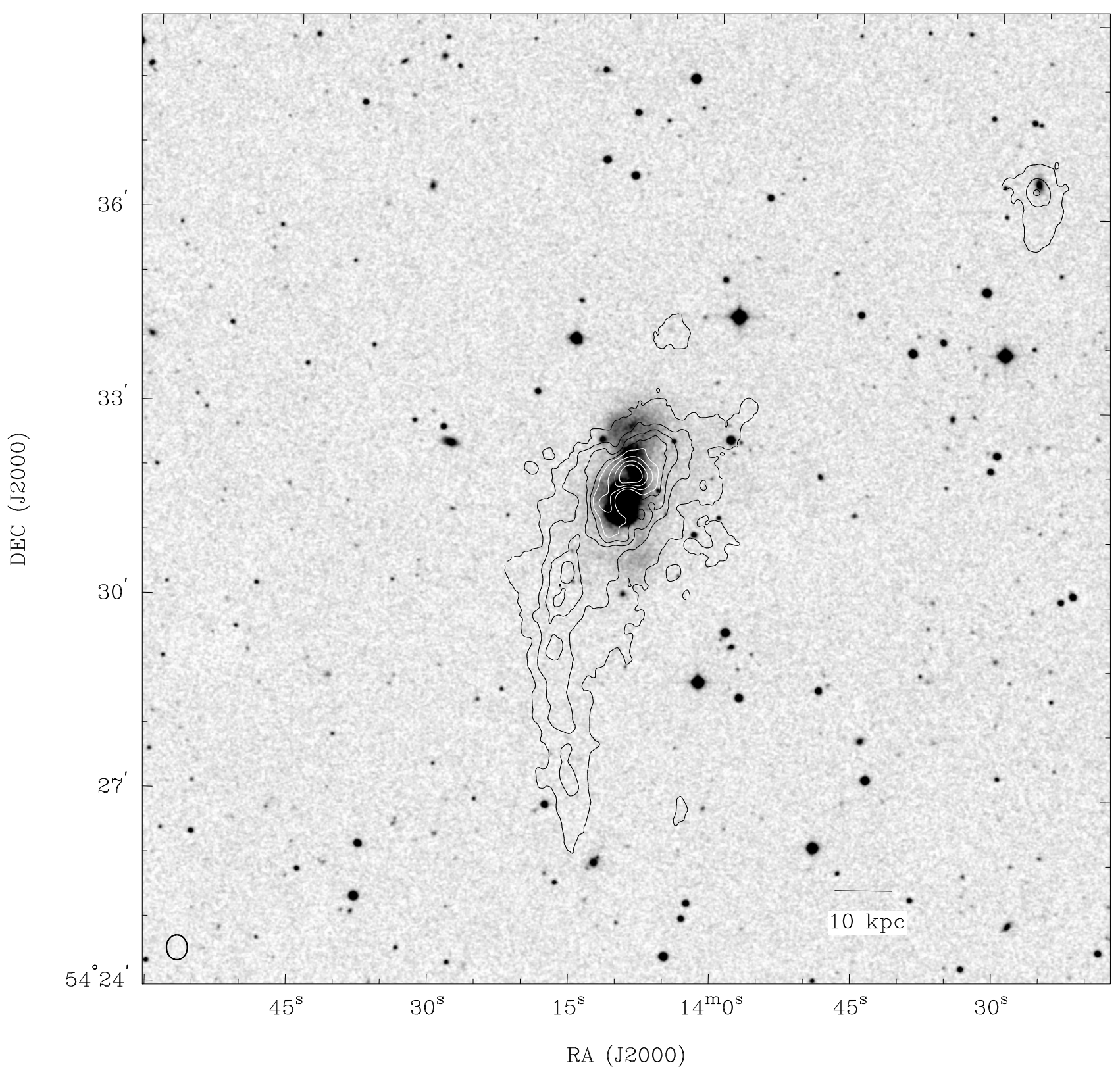

Fig. 2. The HI distribution as contours overlayed on an optical image of the field around NGC 4194 from the Digitized Sky Survey. The contour levels are $0.01,0.05,0.1,0.15,0.2,0.3,0.4,0.5 \mathrm{Jy}$ beam $\mathrm{km} \mathrm{s}^{-1}$. The bar in the lower right corner marks a projected distance of $10 \mathrm{kpc}$, assuming a distance of $39 \mathrm{Mpc}$. The lack of contours at the center of NGC 4194 is due to an absorption source. The beam is shown in the lower left corner.

Table 3. HI properties of the Medusa merger and of the nearby dwarf galaxy SDSS J121326.03+543631.7. The values listed for NGC 4194 include the contribution from the HI tail.

\begin{tabular}{lccc}
\hline \hline HI properties & NGC 4194 & Tail & Nearby dwarf \\
\hline$v_{\text {cent }}\left(\mathrm{km} \mathrm{s}^{-1}\right)$ & 2500 & & 2370 \\
Range $\left(\mathrm{km} \mathrm{s}^{-1}\right)$ & $2400-2580$ & $2470-2520$ & $2350-2390$ \\
Extension $(\mathrm{kpc})$ & 81 & 56 & 14 \\
$F_{\mathrm{HI}}\left(\mathrm{Jy} \mathrm{km} \mathrm{s}^{-1}\right)$ & 5.65 & 2.03 & 0.20 \\
$M_{\mathrm{HI}}\left(10^{9} M_{\odot}\right)$ & 2.0 & 0.7 & 0.07 \\
$M_{\mathrm{HI}} / L_{\mathrm{B}}$ & 0.18 & - & - \\
\hline
\end{tabular}

map. It seems therefore likely that also the high dispersion in $\mathrm{HI}$ is related to the dust lane rather than the starburst region, even though the spatial resolution of the HI presented here is not sufficient for a detailed investigation.

\subsection{2. $\mathrm{HI}$ in absorption toward the center}

HI is seen in absorption against the underlying continuum source of the star forming nucleus. Figure 7 shows the measured spectrum. The multicomponent velocity structure clearly seen in the MERLIN data and discussed by Beswick et al. (2005) is tentatively seen here, too. Our spectrum shows at least two components, one at $\sim 2500 \mathrm{~km} \mathrm{~s}^{-1}$ and one at $\sim 2570 \mathrm{~km} \mathrm{~s}^{-1}$.

\subsubsection{The HI tail}

We detected a single remarkable HI tail going to the South out to $\sim 56 \mathrm{kpc}$ from the center. The HI tail contains several clumps embedded in smooth, diffuse HI emission. Neither in our $V$-band image (which, unfortunately, covers only the upper part of the HI tail) nor in the larger DSS image did we find any optical 

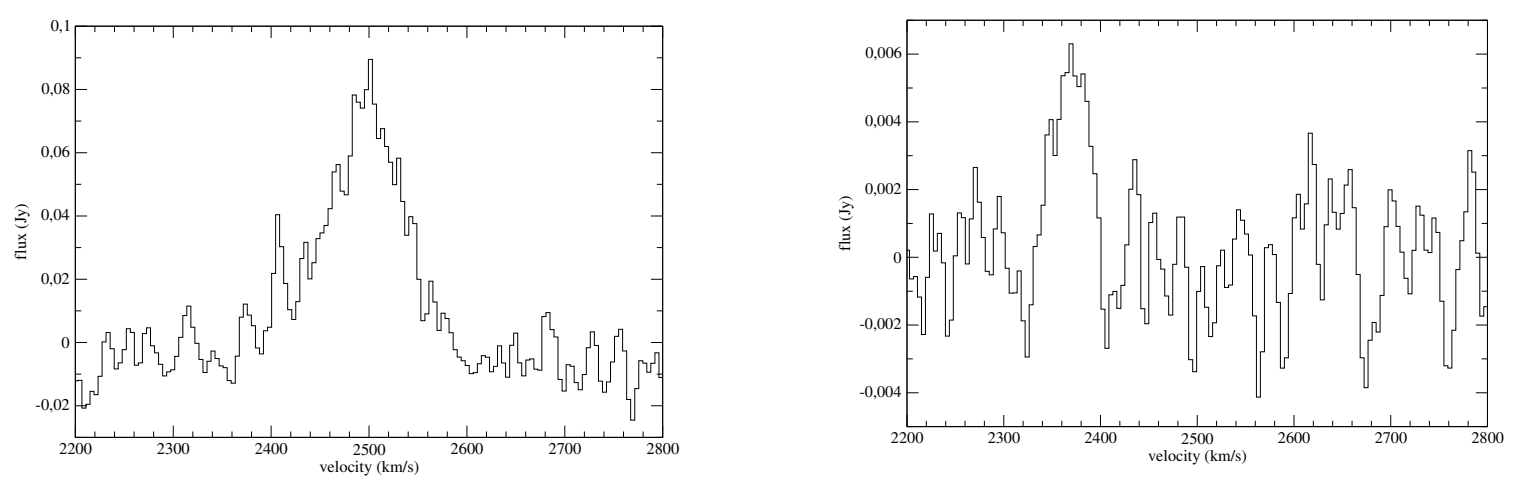

Fig. 3. Integrated HI spectra of NGC 4194 (left) and the companion dwarf galaxy to the North-West (right).

counterpart of the HI clumps. We determined the HI mass in the tail as $\sim 7 \times 10^{8} M_{\odot}$, i.e. $35 \%$ of the total HI mass. The velocity in the tail is smoothly increasing, with the highest velocity of $\sim 2510 \mathrm{~km} \mathrm{~s}^{-1}$ at the tip of the tail. In the velocity dispersion map the smooth behaviour of the tail is found as a continuous but not disturbed increase of dispersion from the tip of the tail towards the galactic body. The dispersion is generally higher at positions of the clumps, especially in two large clumps close to the galaxy's main body.

The lowest velocity $\left(\sim 2455-2480 \mathrm{~km} \mathrm{~s}^{-1}\right)$ is found in the region between the central HI concentration and these two bright clumps, marking the beginning of the tail. In this region, there is a gradient in velocity from north-east to south-west, ending in an area having the lowest velocity in the system southwards of the center. North-west of the two clumps the smooth decrease of velocity is intercepted by a "ridge" of slightly increased velocity. In the velocity dispersion map we see enhanced dispersion there $\left(\sim 35 \mathrm{~km} \mathrm{~s}^{-1}\right.$ compared to $\sim 10 \mathrm{~km} \mathrm{~s}^{-1}$ left and right of this "ridge"), whereas in the HI distribution map, if at all, there seems to be a slight dip in HI intensity associated with this "ridge".

\subsection{4. $\mathrm{H} \mathrm{I}$ in a nearby dwarf galaxy}

We were also able to detect $\mathrm{HI}$ in the dwarf galaxy $\mathrm{J} 121326.03+543631.7$ at a distance of $8.06^{\prime}(91 \mathrm{kpc})$ to the North-West $\left(\alpha=12^{\mathrm{h}} 13^{\mathrm{m}} 26.38^{\mathrm{s}}, \delta=+54^{\circ} 36^{\prime} 31^{\prime \prime}\right)$. Until now, it was not known that NGC 4194 has a companion: our HI measurements show that this dwarf has a central velocity of $2380 \mathrm{~km} \mathrm{~s}^{-1}$, which is only $\sim 120 \mathrm{~km} \mathrm{~s}^{-1}$ lower than that of NGC 4194, thus probably indicating that the two objects are physically connected. The measured HI velocity is in good agreement with its optical velocity of $2436 \mathrm{~km} \mathrm{~s}^{-1}$ (SDSS data release 2, 2004). We carefully checked our HI channel maps for an HI bridge, but even in the naturally weighted maps no connection is visible. Figure 3 shows the integrated HI spectra of NGC 4194 and the nearby galaxy derived from the data cube. With an HI line width of $\sim 60 \mathrm{~km} \mathrm{~s}^{-1}$ and an HI mass of $7 \times$ $10^{7} M_{\odot}$ this dwarf galaxy is significantly smaller than NGC 4194 and thus does not play an important role for the evolution of NGC 4194.

\section{4. $N$-body simulations}

We have performed numerical simulations in order to test whether the current appearance of the Medusa could be due to a gravitational encouter between a gas-rich disk-like galaxy and an elliptical. Our goal was not to reproduce every detail of the Medusa, but rather to see whether a simple type of encounter could generate the observed overall distribution of the stars and the gas, and in particular the following striking patterns: i) the diffuse region of stellar emission to the north (the "hair"); ii) the tangential arcs seen in the stellar distribution to the south; iii) the ca $50 \mathrm{kpc}$ long tail of atomic gas to the south.

\subsection{The model}

We have used numerical simulations to follow the merging process of two galaxies, a gas-rich disk-like galaxy (of spiral type) falling into a larger spherical system (an "elliptical" galaxy).

The spherical galaxy is modeled by a set of collisionless particles distributed according to the Hernquist profile (1990), with a potential of the form $\Phi(r)=-G M /(r+a)$, where $G$ is the gravitational constant, $M$ is the mass, $r$ is the radius and $a$ is a scale length. That model has a simple density profile $\left(\rho \propto r^{-1}(r+a)^{-3}\right)$ and produces a surface density profile in good agreement with the de Vaucouleurs $R^{1 / 4}$ law observed in elliptical galaxies, where $R$ is the projected radius on the sky. Particles are assigned random velocities calculated from the energy distribution function given by Hernquist (1990).

For simplicity, the elliptical galaxy is represented by a single component (collisionless particles in a single Hernquist model) and no distinction is made between stars and dark matter. Observationally, it is difficult to trace the mass distribution in elliptical galaxies out to large radii and although the amount of data is rapidly increasing (e.g., Napolitano et al. 2005) the scatter is large and the fraction of dark matter in ellipticals is still subject to interpretation (e.g., Romanowsky et al. 2003; Dekel et al. 2005). No measurements of the stellar kinematics that would constrain on the mass-to-light ratio exist for the Medusa. In our model, the total mass of the elliptical is four times that of the infalling spiral. It corresponds to $6.66 \times 10^{11} M_{\odot}$, and about $50 \%$ of that mass is contained within a radius of $12 \mathrm{kpc}$. In the following we call the collisionless particles "stars", but it should be kept in mind that some of them, especially at large radii, could be regarded as dark matter particles. Since both the stars and dark matter particles have the same dynamics, having a mix of stars and dark matter woudn't affect the orbit of the infallling companion or the evolution of the system.

The companion galaxy consists of a stellar disk, a gaseous disk, and a dark matter halo. The particles in the disk are distributed in two potentials of the form $\Phi(R, z)=$ $-G M / \sqrt{R^{2}+\left(a+\sqrt{z^{2}+b^{2}}\right)^{2}}$, where $M$ is the mass, $R$ is the radius in the plane of the disk, $z$ is the coordinate perpendicular to the plane, and $a$ and $b$ are scale lengths (Miyamoto \& Nagai 1975). The advantage of that potential is that the scale lengths can be adjusted to represent different types of disks with 

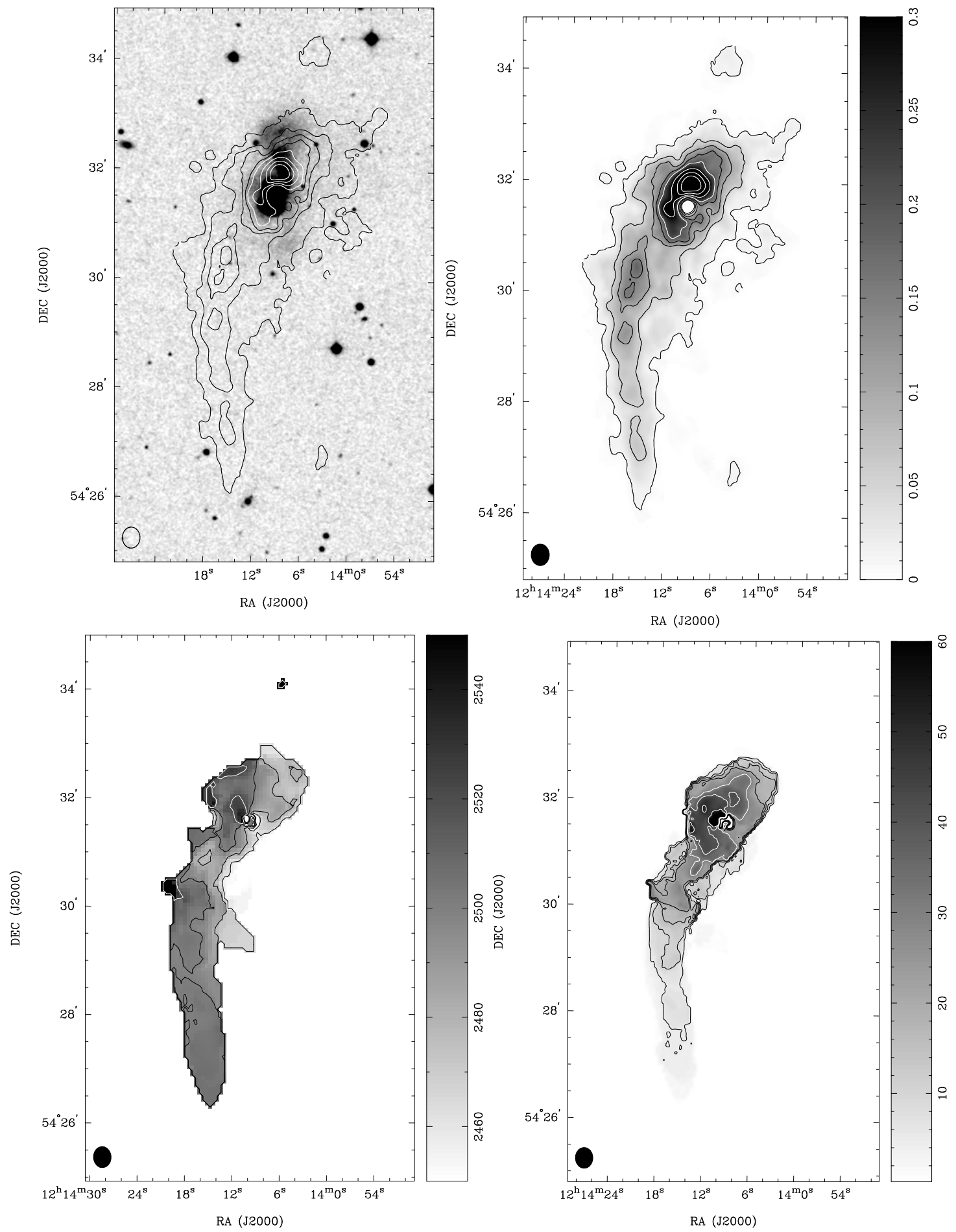

Fig. 4. Maps of the HI distribution, velocity field and velocity dispersion. Top left: HI distribution overlayed on optical image from the Digitized Sky Survey. The contour levels are $0.01,0.05,0.01,0.15,0.2,0.3,0.4,0.5 \mathrm{Jy}$ beam km s${ }^{-1}\left(0.01 \mathrm{Jy} /\right.$ beam km s${ }^{-1}$ corresponds to $\left.2.47 \times 10^{19} \mathrm{~cm}^{-2}\right)$. Top right: HI distribution as map and contours. Levels are the same. The white dot marks the region where HI absorption is found. Bottom left: velocity field, contour levels are from $2460 \mathrm{~km} \mathrm{~s}^{-1}$ to $2600 \mathrm{~km} \mathrm{~s}^{-1}$ in steps of $20 \mathrm{~km} \mathrm{~s}^{-1}$. Bottom right: HI velocity dispersion, contour levels are 5 , $10,15,20,30,40,50 \mathrm{~km} \mathrm{~s}^{-1}$. The synthesised beam is marked in the bottom left corner.

a more or less prominent central bulge. A Hernquist model is used to represent the collisionless halo. Initially, the particles are assigned circular velocities in centrifugal equilibrium with the gravitational potential, and velocity dispersions are given by Toomre's stability criterion (Toomre 1964).
Initial conditions were generated from the distributions described above using Matlab. The evolution of the systems was computed by the programme COCKTAIL (Collisions of Clouds Keep Tree Algorithm in Labor). The collisionless part is the tree algorithm of Barnes \& Hut (1986). The gas is modeled as an 


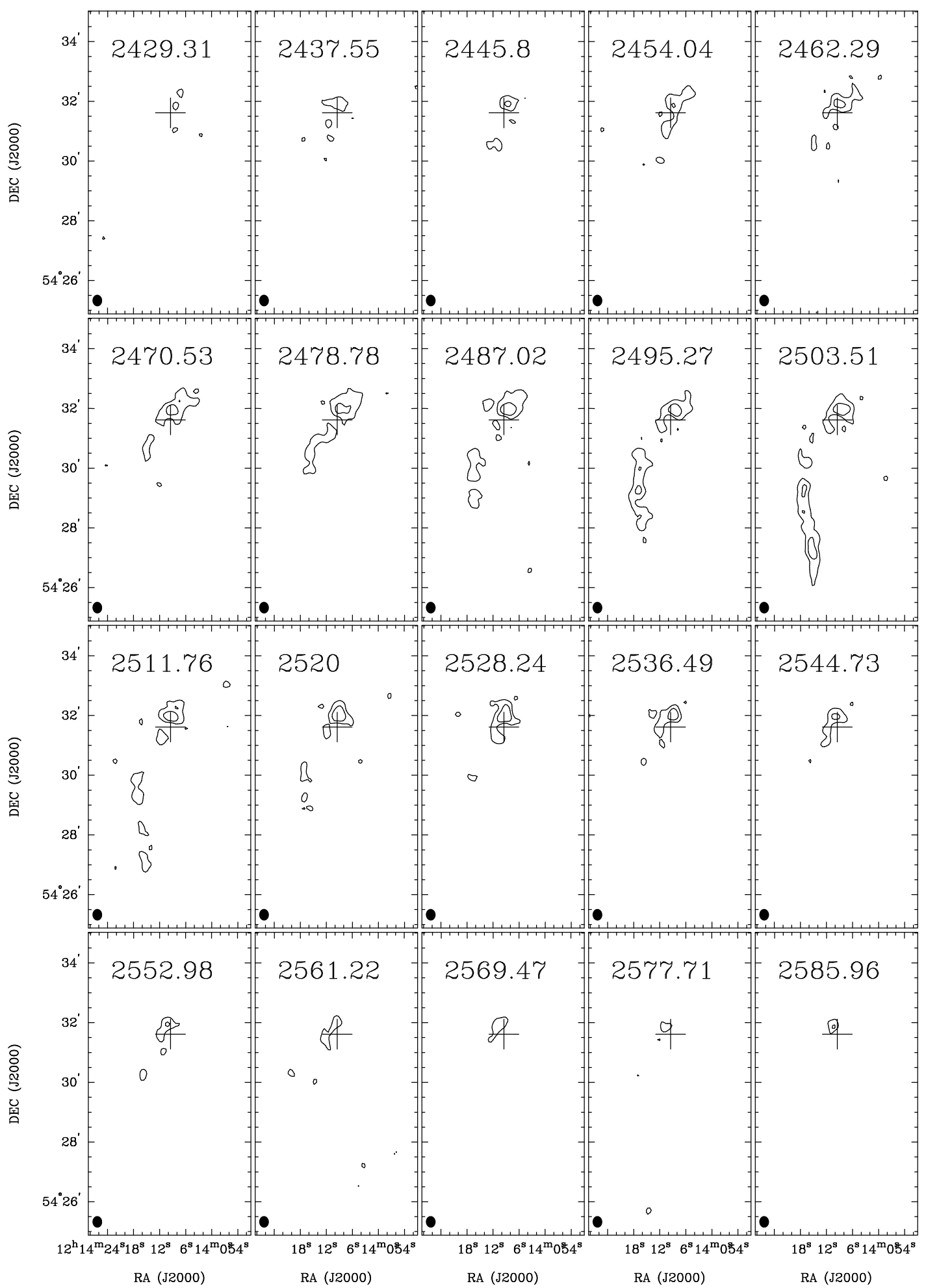

Fig. 5. HI channel maps. The optical center position is marked with a cross. The contour levels are $1,2,5 \times 0.0016 \mathrm{Jy}^{\text {beam }}{ }^{-1}$. The velocity in the Local Standard of Rest is given at the top, the beam is shown at the bottom left corner.

ensemble of clouds, all of the same mass, that dissipate energy through inelastic collisions with each other ("sticky particles", e.g., Combes \& Gerin 1985; Kojima \& Noguchi 1997). The gas accounts for about $10 \%$ of the mass of the stellar disk in the infalling galaxy.

We used a total of 120000 particles: 30000 particles in the elliptical galaxy and 90000 particles in the spiral (40000 in the stellar disk, 20000 in the gaseous disk, 30000 in the dark matter halo).

The parameters of the simulation presented here are listed in Table 4. Setting the units of length and time to $1 \mathrm{kpc}$ and $10^{7}$ years, with the gravitational constant $G$ set to 1 , gives a unit of mass of $2.22 \times 10^{9} M_{\odot}$. We considered encounters of galaxies of different mass ratios and finally selected a mass ratio of $4: 1$, 


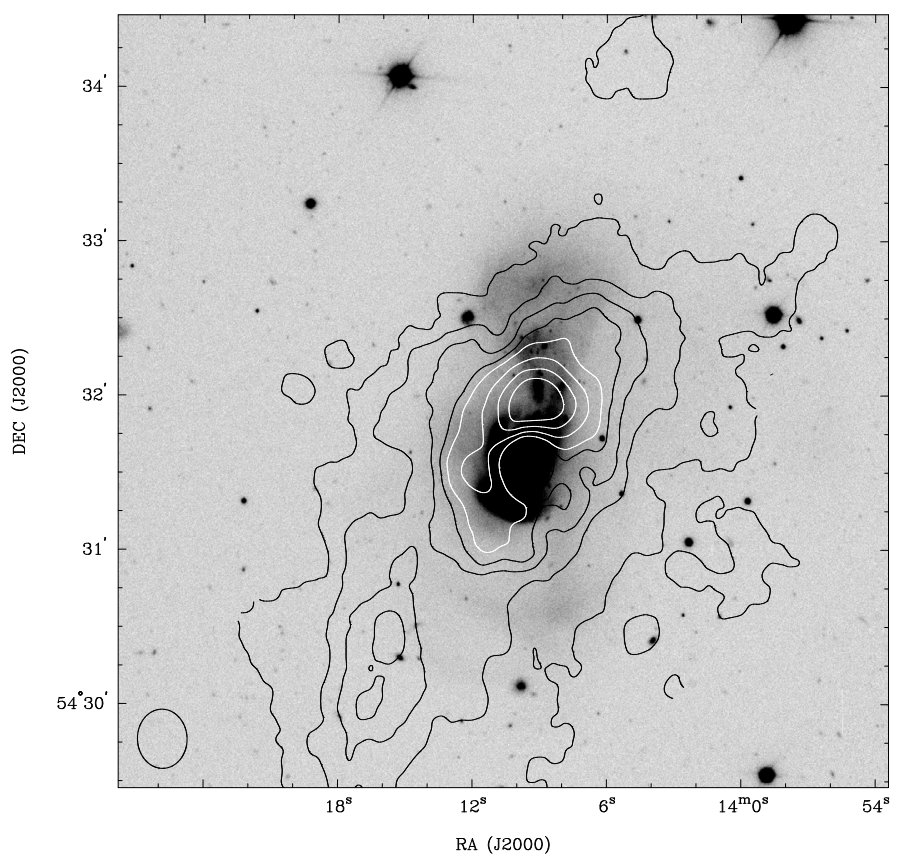

Fig. 6. Contours of the HI intensity map overlayed on the $V$-band image. The optical image covers only the upper part of the HI tail. The contour levels are $0.01,0.05,0.1,0.15,0.2,0.3,0.4,0.5 \mathrm{Jy} \mathrm{beam}^{-1} \mathrm{~km} \mathrm{~s}^{-1}$.

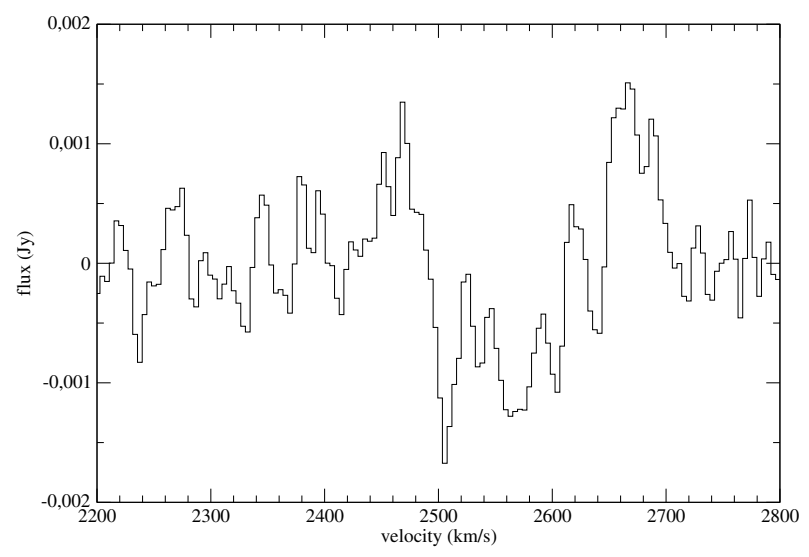

Fig. 7. The HI absorption spectrum toward the center of NGC 4194.

where the more massive galaxy is the elliptical. The initial mass of the gas in the spiral galaxy was set to match the observations of the Medusa, about $4.4 \times 10^{9} M_{\odot}$. The mass of a gas particle is therefore $2.2 \times 10^{5} M_{\odot}$, which is of the order of that a giant molecular cloud. The mass of a star particle in the disk galaxy is $10^{6} M_{\odot}$, and it is about $22 \times 10^{6}$ in the elliptical galaxy. Ideally, all particles of a given type should have a comparable mass to prevent artificial $N$-body scattering (Chandrasekhar 1942). In practice, however, this requirement is difficult to meet because of the limitation on the total number of particles set by an affordable computing time.

Being mainly interested in the kinematical evolution of the stars and the gas in the infalling galaxy (and not in the internal relaxation of the merger remnant, which would be more sensitive to this issue), we used a comparatively larger number of particles to represent the stellar and the gaseous components of the infalling galaxy.

The gas was initially distributed in a Miyamoto-Nagai disk with a scale length twice as large as that of the stellar disk, and the gaseous disk was truncated at an outer radius of $10 \mathrm{kpc}$.
Table 4. Parameters of the simulations, in computing units. The units can be translated in the following physical units: $1 \mathrm{kpc}, 10^{7} \mathrm{yr}$, $100 \mathrm{~km} \mathrm{~s}^{-1}, 2.22 \times 10^{9} M_{\odot}$.

\begin{tabular}{ll}
\hline \hline Mass ratio & $4: 1$ \\
Elliptical: mass and scale length & $M_{\text {ell }}=300, a_{\text {ell }}=5$ \\
initial position and velocity & $x=+10, y=0, z=0$ \\
& $v x=0, v y=0, v z=0$ \\
Disk galaxy: & $M_{\text {stellar disk }}=18, a_{\text {stellar disk }}=1$ \\
mass and scale length & $b_{\text {stellar disk }}=0.1$ \\
& $M_{\text {gaseous disk }}=2, a_{\text {gaseous disk }}=2$ \\
& $b_{\text {gaseous disk }}=0.1$ \\
& $M_{\text {halo }}=55, a_{\text {halo }}=5$ \\
initial position and velocity & $x=-40, y=0, z=0$ \\
& $v x=0, v y=+0.2, v z=0$ \\
\hline
\end{tabular}

The scale length of the elliptical galaxy was calculated from the measured extent of the Medusa at the 25th magnitude/arcsec ${ }^{2}$ isophote: $D_{25} \times d_{25}=2.3 \times 1{ }^{\prime} 6^{\prime}=26 \times 18 \mathrm{kpc}$ at our adopted distance (RC3). This gives a radius to the 25 th isophote of about $11 \mathrm{kpc}$. The scale length of the Hernquist profile is related to the effective radius by the relation $R_{\mathrm{e}}=1.8153 a$ (Hernquist 1990). For elliptical galaxies, $R_{\mathrm{e}}<R_{25}<2 R_{\mathrm{e}}$. We thus took a scale length of $5 \mathrm{kpc}$ for the elliptical galaxy.

The two galaxies were initially separated by $50 \mathrm{kpc}$, and we tested different initial velocities. We obtained a best match for a slightly prograde encounter. In the simulation presented here, the spiral has initially a small positive tangential velocity $\left(20 \mathrm{~km} \mathrm{~s}^{-1}\right)$.

\subsection{Results}

Figures 8 and 9 display the evolution of the stars and the gas as the spiral galaxy falls into the larger elliptical. Tidal distortions appear after closest passage and develop into a tidal tail, made of both stars and gas. The tail lengthens as the core of the infalling galaxy and the larger elliptical galaxy orbit around their center of mass, leading to the eventual merging. Stars and gas are spread in the region diametrally opposite to the tail, forming a diffuse distribution remiscent of the "hair" of the Medusa. As particles from the spiral oscillate, shells form, due to the accumulation of particles at their apocenters.

The central part of the infalling disk galaxy is torn during the interpenetrating encouter with the more massive spherical galaxy. The material that will form the "hair" crosses the center of the spherical galaxy twice and starts expanding into the diffuse "hair" to the North while on the other side material expands into the tidal tail. The core of the infalling galaxy falls back through the center of the spherical a third time, and that is when the simulation resembles the observations most. Stars from the companion oscillate back and forth and shells and loops are seen, but they are transient and their evolution cannot be followed in detail because of the relatively low number of particles that they contain.

Figure 10 shows the distribution of the stars and the gas and the kinematics of the gas at the time when the best match with the observations was found, $T=355 \mathrm{Myr}$ after the beginning of the simulation, after a rotation of $+55^{\circ}$ in the plane of the galaxy. At that time, the length of the tail is about $50 \mathrm{kpc}$, and the kinematics are in reasonable agreement with the observations. Figure 10 can be compared both qualitatively and quantitatively to Fig. 4 that shows the observed counterparts. The observed HI velocity field shows no gradient along the tail, as in the simulation. The velocity dispersion map is also shown. The 

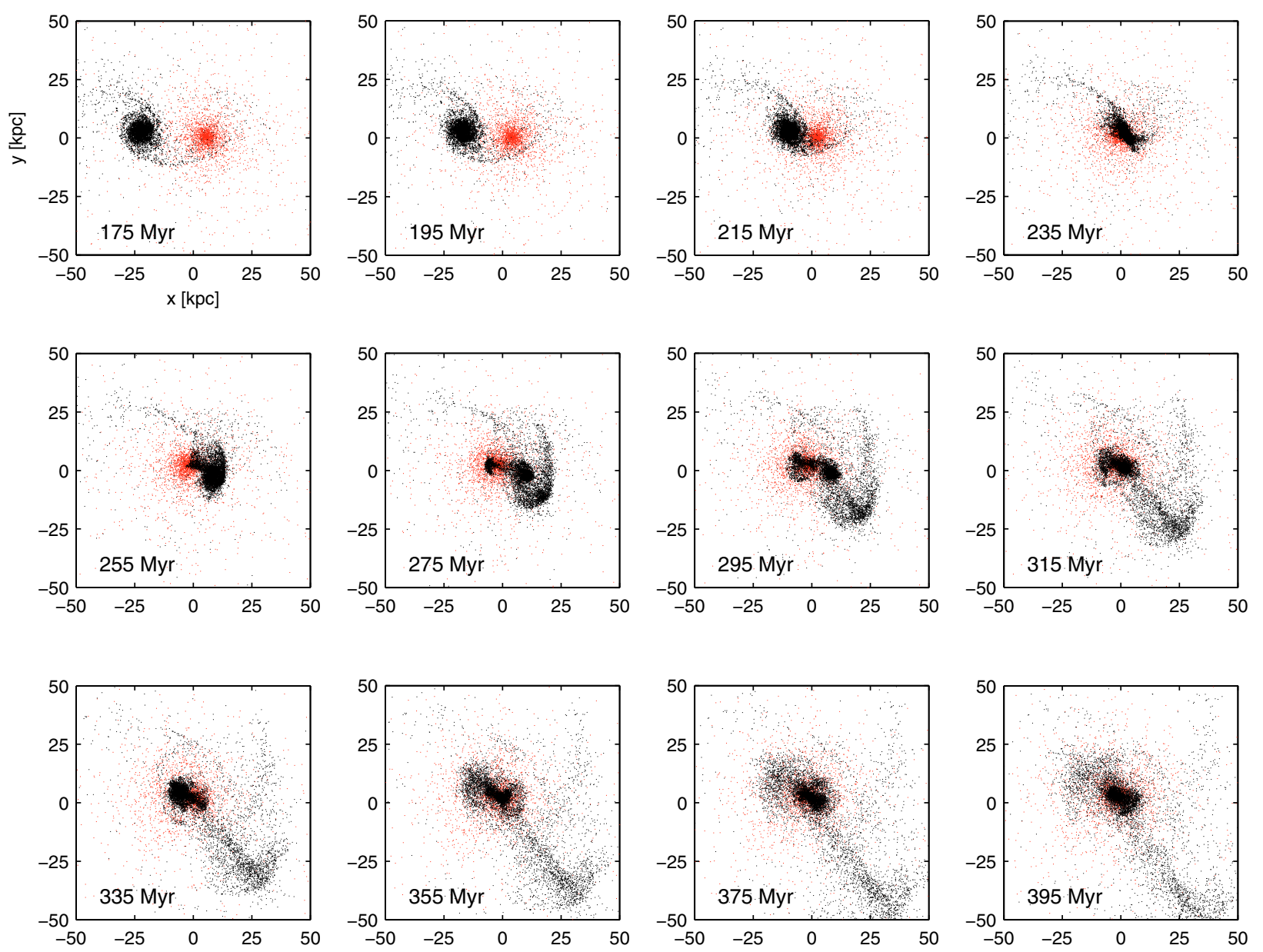

Fig. 8. Evolution of the stars in the simulation. The stars in the elliptical are shown in red, those in the infalling disk galaxy in black.

highest values are found in the central body, whereas the tidal tail shows little velocity dispersion. The isocontours show levels and constant particle density per pixel, where the pixel size of the simulated map is $(1.5 \mathrm{kpc})^{2}$. A density of 100 particles/pixel corresponds to $10^{7} M_{\odot} \mathrm{kpc}^{-2}$, or a column density of hydrogen of $1.25 \times 10^{21} \mathrm{~cm}^{-2}$, which translates into an HI surface brightness of $0.5 \mathrm{Jy} \mathrm{km} \mathrm{s}^{-1}$ beam ${ }^{-1}$. In the simulation, the levels of highest gas density are therefore about twice as high as the highest contour in the observed map. This was to be expected, since our total gas mass is exactly twice as large at the HI mass and includes the molecular component, which is located in the central region of the galaxy. The simulation doesn't treat differently the atomic and the molecular gas. In the tail, the levels are comparable in the simulation and in the observed HI map. Defining the tail as the region with $y<-10 \mathrm{kpc}$ (see Fig. 10), about $30 \%$ of the gas particles and $20 \%$ of the star particles lie in the tail. The gas fraction in the tail is comparable to the observed one. The amount of stars correspond to a surface brightness below the sensitivity of the optical observations.

Let us now examine the kinematics of the gas in the simulation. The particles forming the extended tidal tail to the South and those in the diffuse "hair" to the North can be identified in the simulation by their different kinematics, as illustrated in Fig. 11. The left figure shows the radial oscillations of the gas particles in the infalling galaxy. The orbit crowding resulting in the tidal tail is clearly visible, starting at $T=210 \mathrm{Myr}$. The pattern is reminiscent to that due to radial waves in collisional ring galaxies (e.g. Appleton \& Struck-Marcell 1996;
Horellou \& Combes 2001). Here, however, the phenomenon is different because there is no quasi-perpendicular impact with respect to the plane of the disk galaxy that would cause an expanding ring wave. The crowded particles seen in Fig. 11 are not located at all azimuthal angles, but come from an unwinding arm that forms in the infalling galaxy after the crossing of the elliptical. At $T=355 \mathrm{Myr}$, when the system resembles the Medusa most (the straight vertical line in the figure), most particles at a radius larger than $30 \mathrm{kpc}$ are on their way out, wherease the ones at a smaller radius are falling back toward the galaxy. This is seen in a different way in the figure to the right, which displays the radial velocity of the gas particles versus their radius. This is related to the slope of the curves in the left figure. Particles can be separated into three groups: the tail, expanding beyond $r \simeq 30 \mathrm{kpc}\left(v_{\mathrm{rad}}>0\right)$, the "hair", expanding beyond $r \simeq 12 \mathrm{kpc}$, and the rest, mostly located within $r \leq 10 \mathrm{kpc}$.

Since the different components have clear different kinematics, we would like to be able to identify them in the observed HI data. Unfortunately, since the system is seen very close to face-on, the kinematical signatures which appear in the radial plots are not present when we examine the line-of-sight velocity components, which are displayed in Fig. 12 for both the simulation at $T=355 \mathrm{Myr}$ (left) and the observations. The two plots are similar, displaying the gas tail extending to about $60 \mathrm{kpc}$ with a small and constant velocity dispersion as a function of radius. The simulation shows more gas in the center than the observation which is sensitive to the atomic gas only, whereas the initial amount of gas in the simulation was taken to match both 

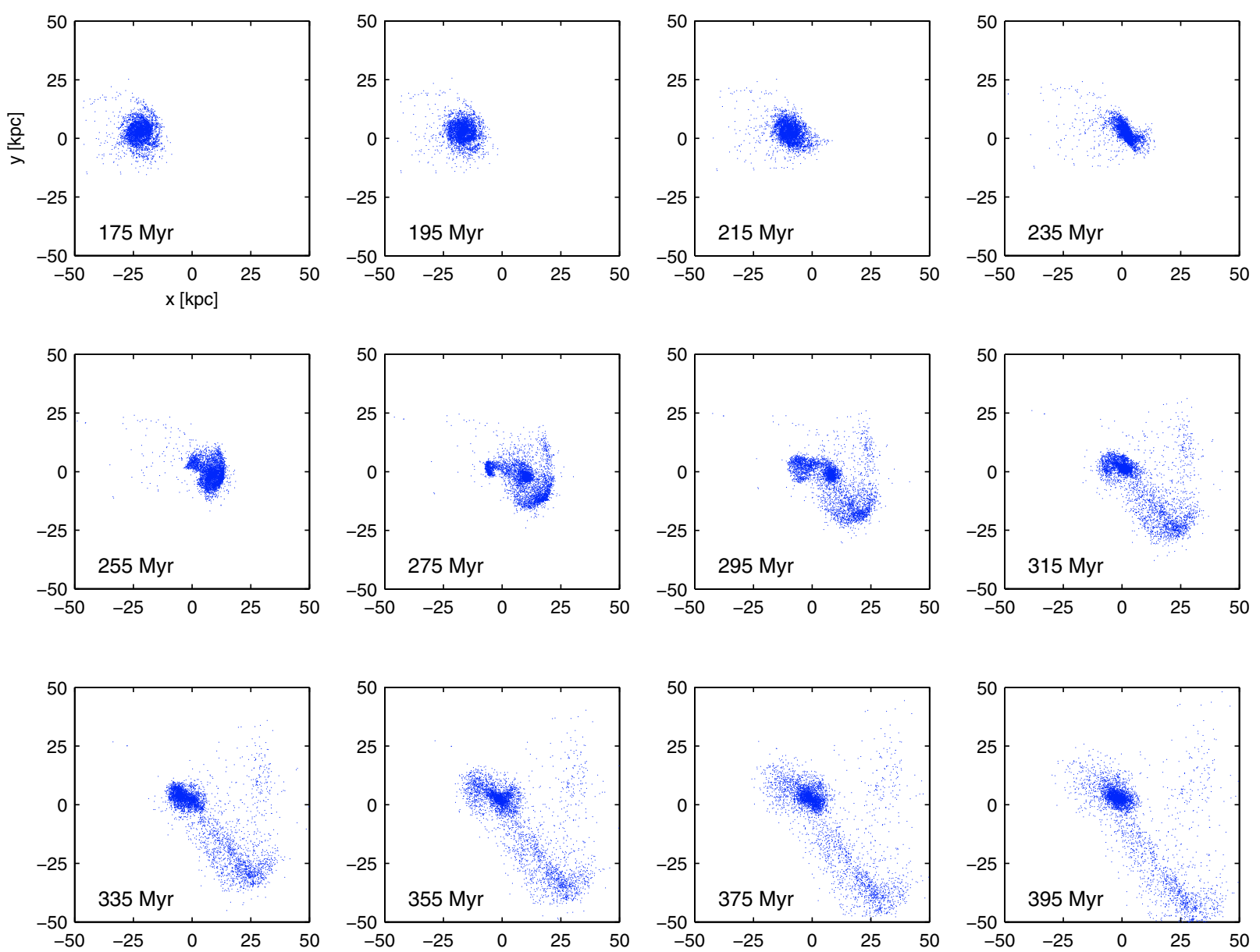

Fig. 9. Evolution of the gas in the simulation.

Table 5. The measured continuum flux, star formation rates based on continuum and FIR emission, respectively, and $q$ parameter.

\begin{tabular}{lc}
\hline \hline Properties & \\
\hline $20 \mathrm{~cm}(\mathrm{Jy})$ & 0.108 \\
$S F R_{20 \mathrm{~cm}}\left(M_{\odot} \mathrm{yr}^{-1}\right)$ & 22.8 \\
$S F R_{\mathrm{FIR}}\left(M_{\odot} \mathrm{yr}^{-1}\right)$ & 9.5 \\
$q$ & 2.5 \\
\hline
\end{tabular}

the molecular component (located in the inner part of the galaxy and traced by $\mathrm{CO}$ observations) and the atomic component.

\section{Discussion}

\subsection{Star formation rate}

We followed Condon (1992) and Haarsma et al. (2000) and used the relation below to calculate the $S F R$ from the $20 \mathrm{~cm}$ continuum flux:

$\operatorname{SFR}\left(M_{\odot} \mathrm{yr}^{-1}\right)=0.14 D^{2} F_{20 \mathrm{~cm}}$

where $D$ is the distance in $\mathrm{Mpc}$ and $F_{20 \mathrm{~cm}}$ is the 20-cm radio continuum flux density in Jy. The results are given in Table 5. We derived a SFR which is comparable to the results of Condon et al. (1990), who measured a flux of $122 \mathrm{mJy}$ with the VLA and thus determined the $S F R$ in the Medusa as $\sim 20 M_{\odot} \mathrm{yr}^{-1}$.

Table 5 also gives the SFR estimated from the FIR luminosity:

$\operatorname{SFR}\left(M_{\odot} \mathrm{yr}^{-1}\right)=0.17 L_{\mathrm{FIR}}$ following Kennicutt (1998), with $L_{\mathrm{FIR}}$ in units of $10^{9} L_{\odot}$. As a reference for the IRAS $60 \mu \mathrm{m}$ and $100 \mu \mathrm{m}$ flux densities in Table 1 we used the IRAS Faint Source Catalog (Moshir et al. 1990). The relation between the $S F R$ estimated from the $20 \mathrm{~cm}$ flux and the FIR fluxes can be expressed by the $q$ parameter defined by Helou et al. (1985), which is the logarithmic ratio of FIR to radio flux. Helou et al. (1985) derived a mean value of 2.3 for disk galaxies, independent of their star formation activity. Although NGC 4194 is a merging galaxy, it conforms to this value which is a good indicator that the continuum emission is dominated by emission from the starburst. Helou et al. (1985) mention that even galaxies with a strong nuclear starburst, as Arp 220, are in agreement with this correlation of the FIR and radio flux, however within a higher scatter. Arp 220, e.g., has a $q$ value of 2.65, whereas other prominent ULIRGs like Arp 299 and Mrk 231 have a value of 2.1.

Our results are in good agreement with the investigations of Weistrop et al. (2004), who used the UV flux of the central star forming knots to estimate the star formation rate. They estimate a $S F R$ within the knots of 5-6 $M_{\odot} \mathrm{yr}^{-1}$ and suggest an overall SFR of up to $30 M_{\odot} \mathrm{yr}^{-1}$.

The continuum source was studied recently by Beswick et al. (2005) with sub-arcsecond resolution MERLIN data. They found a pair of continuum sources separated $\sim 0.35^{\prime \prime}$, thus unresolved in our image. They derived a $S F R$ of $6 M_{\odot} \mathrm{yr}^{-1}$, in agreement with the results of Weistrop et al. (2004), but they also pointed out that they only measured the star formation rate in the very center of NGC 4194. 

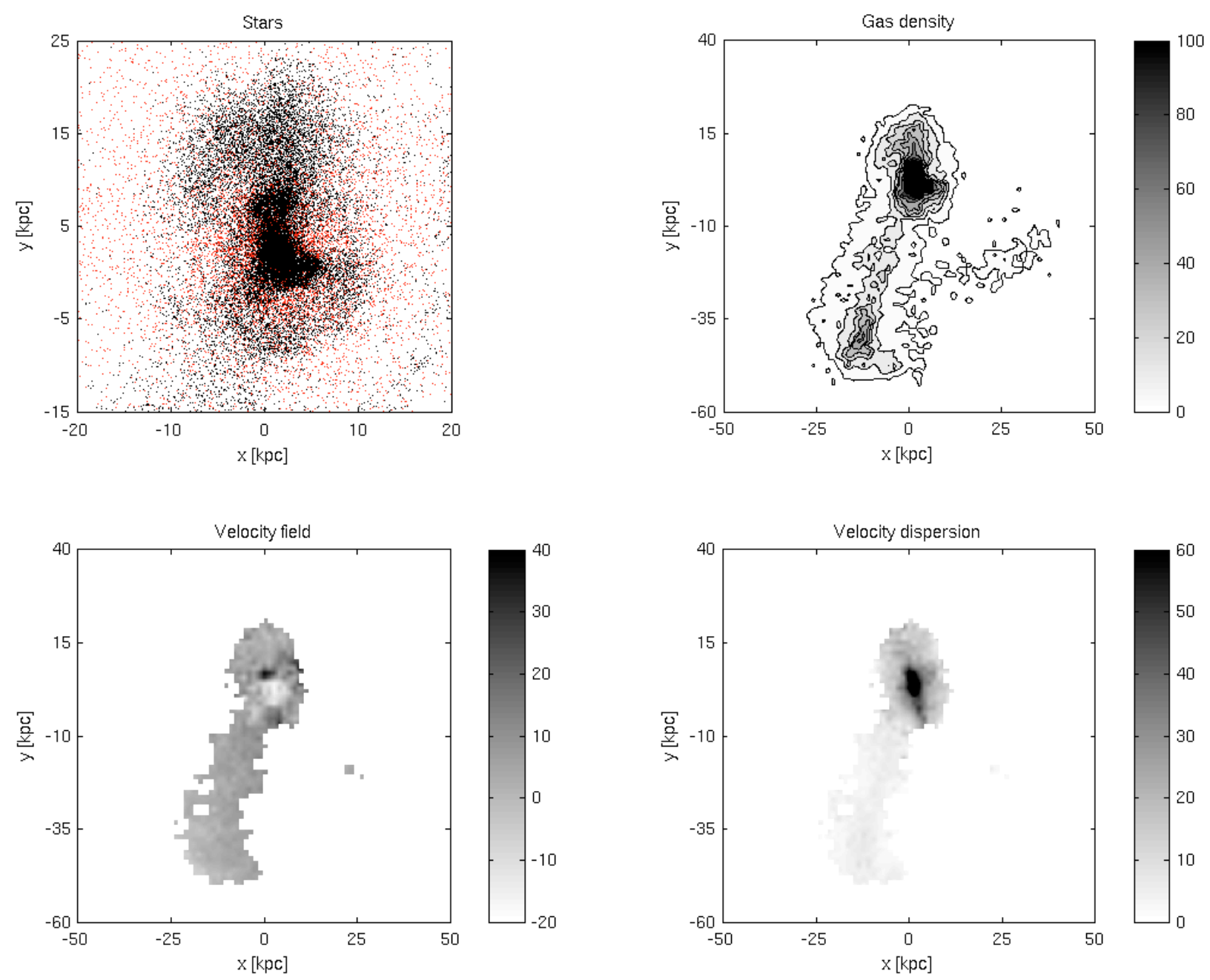

Fig. 10. View at a time corresponding to $T=355 \mathrm{Myr}$ after the beginning of the simulation, when the system resembles the Medusa most. The orbital plane (which is the same as the plane of the disk of the infalling galaxy) also coincides with the plane of the sky. The system was rotated by $55^{\circ}$ compared to the previous figures. a) Distribution of the stars. b) Distribution of the gas density. The grey scale shows the number of particles per $1.5 \mathrm{kpc}^{2}$ pixel. c) Velocity field of the gas. The grey scale shows the range of velocities in $\mathrm{km} \mathrm{s}^{-1}$. d) Map of the velocity dispersion of the gas. The grey scale shows the range of velocity dispersions in $\mathrm{km} \mathrm{s}^{-1}$.

Our measured continuum source size of $1.2 \mathrm{kpc}$ is only slightly smaller than the star forming region of $\sim 2 \mathrm{kpc}$, as determined by optical and CO observations (Armus et al. 1990; Aalto \& Hüttemeister 2000).

\subsection{The merger remnant}

Our simulations strongly support the hypothesis that the Medusa is the result of a merger between a gas-rich disk galaxy and a larger elliptical, with a mass ratio of about 4 . We have tested a wide range of parameters, and the constraints placed by the observations of the HI tail (its length and kinematics) and the optical appearance of the Medusa made it possible to reduce considerably the parameter space. The encounter that we finally selected is slightly prograde, in the sense that the orbital plane coincides with the plane of the infalling disk galaxy, but the tangential velocity component is low $-20 \mathrm{~km} \mathrm{~s}^{-1}$ - and the infalling galaxy acquires largely radial motion as it falls through the larger elliptical. The two galaxies interpenetrate and the central part of the disk galaxy passes through the center of the elliptical twice, forming the diffuse "hair" on one side. Our simulations are similar to those of Kojima \& Noguchi (1997) who investigated the formation of shells using a model similar to ours, where the gas is represented by "sticky" particles. (Their model, however, included also star formation based on the local gas density and gas consumption). They showed that radial and slightly retrograde mergers lead to the formation of long-lived ( $>1$ Gyr) shells, and that star formation is significantly reduced because the gas is scattered in tidal debris. We have observed similar effects in some of the simulations that we have run. We believe that the Medusa is due to a different type of encounter, namely a slightly prograde one, also discussed by Kojima \& Noguchi (1997), that leads to both the formation of an extended gas-rich tidal tail and to the infall of gas toward the center, where a moderate starburst is observed. Although the overall distribution of the stars and the gas in the simulation is similar, some differences are seen. In low-density regions, the gas behaves like the collisionless stellar component. During the interpenetrating encounter, the rate of collisions between the gas clouds increase, which lead to a loss of energy and angular momentum and an infall of gas toward the center; the gas and the stars have a different initial radial distribution, with the gas being located more in the outer part with a larger angular momentum and lower binding energy. The gas is therefore more susceptible than the stars to be expelled into extended tidal tails. 

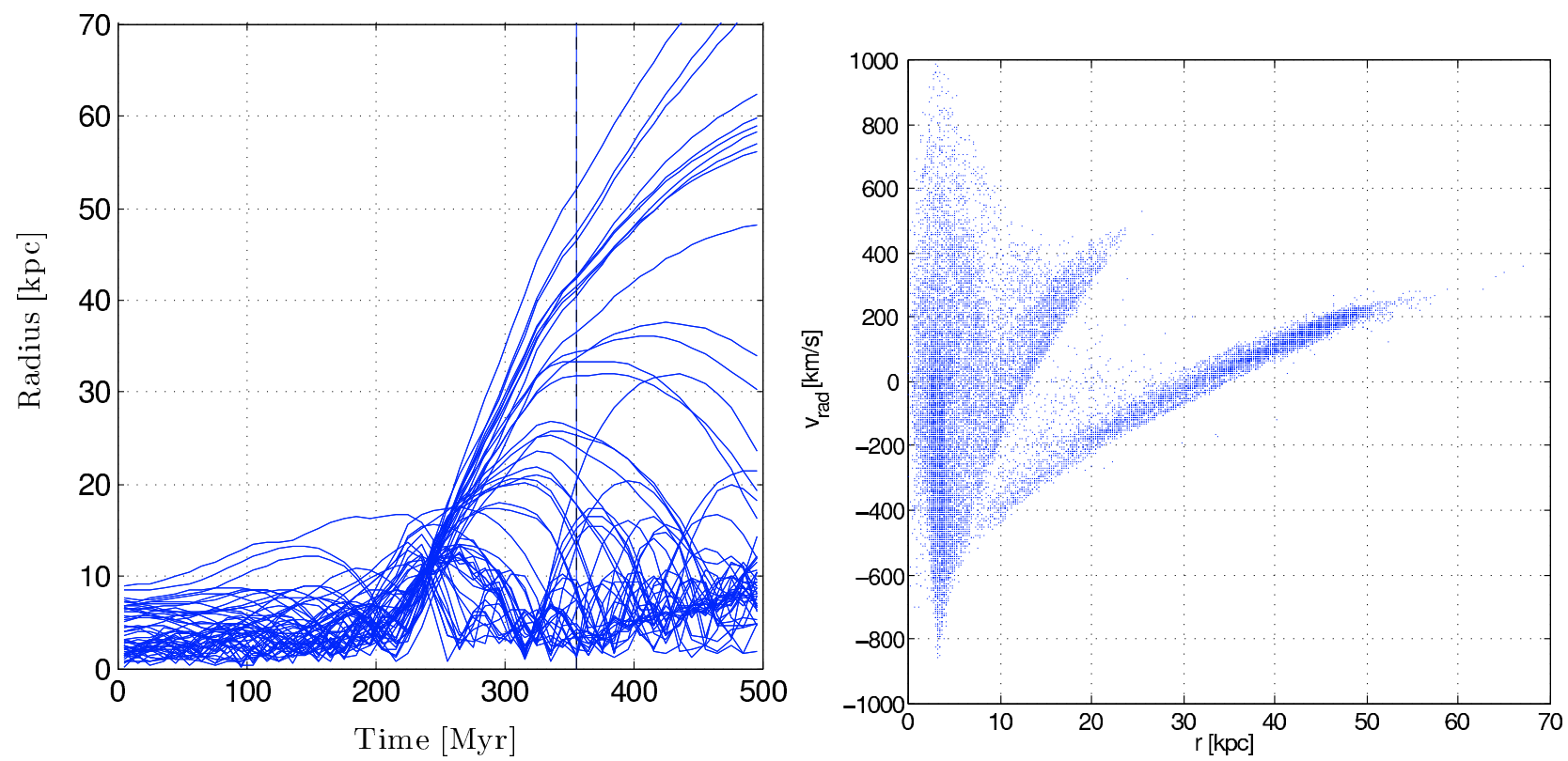

Fig. 11. Kinematics of the gas in the simulation. Left: temporal evolution of the radial distribution of the gas particles in the infalling galaxy. The vertical line at $T=355$ Myr indicates the time when the system resembles the Medusa most. Right: plot at $T=355$ Myr of the radial velocity of the gas particles versus their distance from the center of mass of the infalling galaxy. The "hair" and the tail appear as distinct kinematical features.
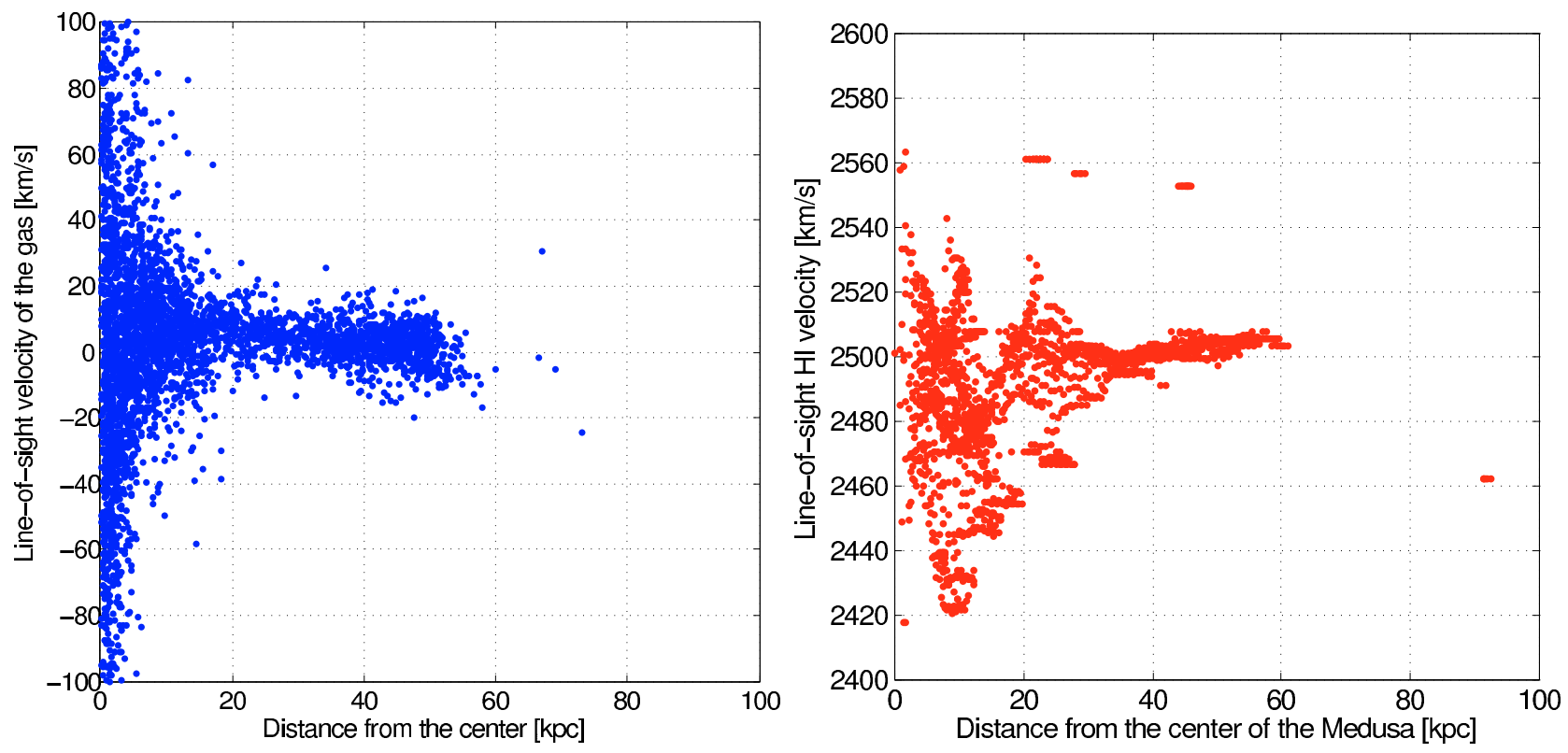

Fig. 12. Line-of-sight velocity of the gas versus distance from a center in the simulation (left) and in the observations (right).

Hibbard et al. (2000) studied some examples of diskdisk mergers which show anticorrelations between stellar and gaseous tidal features. They discuss various possibilities for these differences, including ram pressure stripping, photoionisation, dust obscuration and different origins in the progenitor galaxies. Although possibly at least several scenarios may play a role at some point, the lack of starlight associated with the large HI tail found in NGC 4194 can be easiest explained by the original position of the $\mathrm{HI}$ in the progenitor.

\section{Conclusions}

We have presented new optical, radio continuum and HI line observations of the Medusa galaxy and numerical $N$-body simulations of the merger of a small gas-rich disk galaxy with a larger elliptical galaxy. The optical observations consist of a deep $V$-band image of the distribution of the stars in the Medusa, and the radio observations of maps of the $20 \mathrm{~cm}$ radio continuum emission and of the large-scale distribution and kinematics of the atomic hydrogen gas. The simulations are completely selfconsistent with a total of 120000 particles. The gas in the infalling disk galaxy is represented by an ensemble of clouds, all of the same mass, that can dissipate energy in inelastic collisions with each other.

1. The HI observations revealed the presence of a single HI tail to the South, extending out to a projected distance of $4.95^{\prime}$, or $56 \mathrm{kpc}$ from the center of the Medusa. The tail contains $\sim 7 \times 10^{8} M_{\odot}$ of HI and has no detected optical counterpart. 
No significant velocity gradient is observed along the tail, indicating that it is seen almost face-on.

2. HI was detected in a dwarf galaxy identified at a projected distance of $91 \mathrm{kpc}$ to the North-West of NGC 4194. Its systemic velocity of $2380 \mathrm{~km} \mathrm{~s}^{-1}$ is only $120 \mathrm{~km} \mathrm{~s}^{-1}$ lower than that of the Medusa. This dwarf galaxy harbours $\sim 7 \times 10^{7} M_{\odot}$ of HI. No connection, neither in the optical nor the HI could be found between the dwarf galaxy and NGC 4194 and it is unlikely that it has had an influence on the dynamical evolution of the Medusa.

3. We found a large misalignment between optical and gaseous tidal features. The diffuse optical tail is situated on the opposite side of the HI tail, the optical shells do not seem to be connected to the HI tail. Although the shells are within the region of the HI tail, no connection is apparent neither in morphology nor in velocity distribution.

4. We estimated a $S F R$ of $23 M_{\odot} \mathrm{yr}^{-1}$ based on the $20 \mathrm{~cm}$ continuum flux density, and of $10 M_{\odot} \mathrm{yr}^{-1}$ from the FIR fluxes. This is consistent with a rather intense ongoing burst of star formation in the Medusa, in agreement with the optical and UV observations of Weistrop et al. (2004).

5. HI was detected in absorption toward the radio continuum in the center.

6. The simulations of a slightly prograde merger between a gasrich disk galaxy with a total mass four times less than that of the elliptical give the best match to the observations. A extended single tidal tail develops in the outer part while the small galaxy falls in and oscillates around the center of the large elliptical, creating shell-like structures around the elliptical, best visible in the stars. The overall distribution of the gas is similar to that of the stars, although more gas falls toward the center because of the dissipative nature and more gas is found of the extended tail because of the initially more extended distribution of the gas. The tail is mostly formed by material of the outer HI disk of the progenitor spiral galaxy, and the surface brightness of the stellar material in the tail is low.

Acknowledgements. We thank the referee, Frédéric Bournaud, for a prompt and useful report. We thank the staff at the Westerbork Radio Synthesis Telescope for assistance during the observations, Tom Oosterloo and Gustaf Rydbeck for helping with some aspects of the data processing. We are grateful to Alessandro Romeo for useful discussions, to Joshua Barnes and to Piet Hut for making the treecode public and to Françoise Combes for providing us with a version of COCKTAIL. The Graduiertenkolleg 787 and the Swedish Research Council are acknowledged for funding support. This research has made use of NASA's Astrophysics Data System Bibliographic Services and of the NASA/IPAC Extragalactic Database (NED) which is operated by the Jet Propulsion Laboratory, California Institute of Technology, under contract with the National Aeronautics and Space Administration.

\section{References}

Aalto, S., \& Hüttemeister, S. 2000, A\&A, 362, 42

Aalto, S., Hüttemeister, S., \& Polatidis, A. G. 2001, A\&A, 372, L29

Appleton, P. N., \& Struck-Marcell, C. 1996, Fundamentals of Cosmic Physics, 16,111

Armus, L., Heckman, T. M., \& Miley, G. K. 1990, ApJ, 364, 471

Barnes, J., \& Hut, P. 1986, Nature, 324, 446

Barnes, J. E., \& Hernquist, L. E. 1991, ApJ, 370, L65

Barnes, J. E., \& Hernquist, L. 1996, ApJ, 471, 115

Beswick, R. J., Aalto, S., Pedlar, A., \& Hüttemeister, S. 2005, A\&A, 444, 791

Bournaud, F., Jog, C. J., \& Combes, F. 2005, A\&A, 437, 69

Chandrasekhar, S. 1942, Principles of stellar dynamics, Physical Sciences Data

Combes, F., \& Gerin, M. 1985, A\&A, 150, 327

Condon, J. J. 1992, ARA\&A, 30, 575

Condon, J. J., Helou, G., Sanders, D. B., \& Soifer, B. T. 1990, ApJS, 73, 359

Cox, T. J., Jonsson, P., Somerville, R. S., Primack, J. R., \& Dekel, A. 2008, MNRAS, 384, 386

Dekel, A., Stoehr, F., Mamon, G. A., et al. 2005, Nature, 437, 707

Haarsma, D. B., Partridge, R. B., Windhorst, R. A., \& Richards, E. A. 2000, ApJ, 544,641

Hancock, M., Weistrop, D., Nelson, C. H., \& Kaiser, M. E. 2006, AJ, 131, 282

Helou, G., Soifer, B. T., \& Rowan-Robinson, M. 1985, ApJ, 298, L7

Hernquist, L. 1990, ApJ, 356, 359

Hibbard, J. E., Vacca, W. D., \& Yun, M. S. 2000, AJ, 119, 1130

Horellou, C., \& Combes, F. 2001, Ap\&SS, 276, 1141

Jütte, E., Jütte, M., Hüttemeister, S., \& Aalto, S. 2008, in prep.

Kennicutt, R. C. 1998, ARA\&A, 36, 189

Kojima, M., \& Noguchi, M. 1997, ApJ, 481, 132

Martig, M., \& Bournaud, F. 2008, MNRAS, 385, L38

Mihos, J. C., \& Hernquist, L. 1996, ApJ, 464, 641

Miyamoto, M., \& Nagai, R. 1975, PASJ, 27, 533

Moshir, M., et al. 1990, in IRAS Faint Source Catalogue, version 2.0, 0

Napolitano, N. R., Capaccioli, M., Romanowsky, A. J., et al. 2005, MNRAS, 357,691

Prestwich, A. H., Joseph, R. D., \& Wright, G. S. 1994, ApJ, 422, 73

Romanowsky, A. J., Douglas, N. G., Arnaboldi, M., et al. 2003, Science, 301, 1696

Sanders, D. B., \& Mirabel, I. F. 1996, ARA\&A, 34, 749

Sanders, D. B., Mazzarella, J. M., Kim, D.-C., Surace, J. A., \& Soifer, B. T. 2003, AJ, 126, 1607

Thuan, T. X., \& Martin, G. E. 1981, ApJ, 247, 823

Toomre, A. 1964, ApJ, 139, 1217

Weistrop, D., Eggers, D., Hancock, M., et al. 2004, AJ, 127, 1360 